УДК 519.214.5

\title{
О ЧИСЛЕ ПОЯВЛЕНИЙ ЗНАКОВ \\ В МУЛЬТИЦИКЛИЧЕСКОЙ СЛУЧАЙНОЙ ПОСЛЕДОВАТЕЛЬНОСТИ ПО МОДУЛЮ 4 С М-ЗАВИСИМЫМИ ЗНАКАМИ
}

\author{
Меженная Н.М.
}

ГОУ ВПО «Московский государственный технический университет им. Н.Э. Баумана», Москва, e-mail: natalia.mezhennaya@gmail.com

\begin{abstract}
В работе изучаются свойства мультициклической случайной последовательности по модулю 4, образованной $r$ регистрами. Если регистры независимы между собой и заполнены независимыми случайными величинами регистр, то мультициклическая случайная последовательность представляет собой математическую модель выходной последовательности генератора Пола (см. [6]). В работе изучены свойства мультициклической последовательности, когда регистры независимы между собой, но случайные величины в каждом регистре m-зависимы по кругу. Для случайного вектора из чисел появлений знаков на полном цикле мультициклической последовательности по модулю 4 получен явный вид вектора средних и ковариационной матрицы. Доказана многомерная предельная теорема нормального типа для указанного вектора в случае, когда длины регистров стремятся к бесконечности, а их число г остается фиксированным. Работа выполнена при финансовой поддержке Министерства образования и науки РФ (тема 1.2640.2014).
\end{abstract}

Ключевые слова: мультициклическая последовательность, генератор Пола, m-зависимые случайные величины, нормальная предельная теорема, устойчивость распределения

\section{ON THE NUMBER OF OCCURENCES OF CHARACTERS IN MULTICYCLIC RANDOM SEQUENCE MODULO 4 WITH M-DEPENDENT ITEMS}

\author{
Mezhennaya N.M.
}

Bauman Moscow State Technical University, Moscow, e-mail: natalia.mezhennaya@gmail.com

\begin{abstract}
The work is devoted to studying of properties of multicyclic random sequence modulo 4 generated by $r$ registers. If registers are independent of one another and filled by independent random variables the multicyclic random sequence performs a mathematical model of output sequence of Pohl generator (see [6]). In the paper we studied the properties of multicyclic sequence when the registers are independent of one another but the random variables in every register are circle-wise $\mathrm{m}$-dependent. We devoted the explicit form of vector of means and covariance matrix for the random vector consisted of the numbers of occurrences of characters on the complete cycle of multicyclic sequence modulo 4 . We proved multivariate limit theorem of normal type for the random vector in the case when registers lengths tends to infinity and the number of registers $r$ remains fixed. The work is supported by The Ministry of Education and Science of the Russian Federation (project 1.2640.2014).
\end{abstract} Keywords: multicyclic sequence, Pohl generator, m-dependent random variables, normal limit theorem, robustness of
distribution

Пусть $n_{1}, \ldots, n_{r} \geq 2$ - взаимно простые натуральные числа, $X^{(j)}=\left(X_{0}^{(j)}, \ldots, X_{n_{-}-1}^{(j)}\right)$, $j=1, \ldots, r, \quad$ - наборы случайных величин, распределенных равномерно на множестве вычетов по модулю $M$. Рассмотрим последовательность, построенную по правилу

$$
Z_{t}=\sum_{j=1}^{r} X_{t\left(n_{j}\right)}^{(j)} \bmod M,
$$

где $t\left(n_{j}\right)=t \bmod n_{j}$. Ее принято называть случайной мультициклической последовательностью. Величину $T=n_{1} \ldots n_{r}$ принято называть полным циклом мультициклической последовательности .

Если наборы $X^{(1)}, \ldots, X^{(r)}$ независимы, а случайные величины $X_{0}^{(j)}, \ldots, X_{n_{j}-1}^{(j)}$, образующие $j$-й набор, независимы и распределены равномерно на множестве вычетов по модулю $M$ последовательность вида представляет собой математическую модель выходной последовательности ге- нератора Пола (см. [6]), в которой наборы $X^{(1)}, \ldots, X^{(r)}$ представляют собой заполнения регистров длин $n_{1}, \ldots, n_{r}$. Эта модель используется для изучения статистических свойств выходной последовательности этого генератора.

В работе [1] был исследован случай мультициклической последовательности по модулю 2 с независимыми заполнениями внутри каждого регистра. В частности, получено предельное поведение для числа единиц на цикле мультициклической последовательности. В работе [5] проведено исследование устойчивости свойств полученных асимптотических распределений для числа единиц в случае, когда $M=2$ и заполнения регистров могут быть зависимы между собой. Свойства мультициклической последовательности при $M=4$ и независимых равновероятных знаках в регистрах были исследованы в работе [2]. Получено совместное предельное распределение чи- 
сел появлений знаков в мультициклической последовательности, когда длины регистров стремятся к бесконечности.

Настоящая работа посвящена изучению свойств мультициклической последовательности по модулю $M=4$ вида длины $T$, когда случайные векторы $X^{(1)}, \ldots, X^{(r)}$ независимы между собой, но случайные величины $X_{0}^{(j)}, \ldots, X_{n_{j}-1}^{(j)}$, образующий $j$-й вектор, зависимы.

\section{Предельная теорема}

Сформулируем 3 условия:

1. Пусть $X^{(j)}=\left(X_{0}^{(j)}, \ldots, X_{n_{j}-1}^{(j)}\right)$, $j=1, \ldots, r$, независимые в совокупности случайные векторы, компоненты которых имеют равномерное одномерное распределение

$$
\begin{gathered}
\mathbf{P}\left\{X_{k}^{(j)}=2 a_{2}+a_{1}\right\}=\frac{1}{4}, \\
a_{1}, a_{2} \in\{0,1\}, k=0, \ldots, \\
n_{j}-1, j=1, \ldots, r .
\end{gathered}
$$

2. Пусть при каждом $ј$ случайные величины $X_{0}^{(j)}, \ldots, X_{n_{j}-1}^{(j)} m$ - зависимы по кругу, т.е. наборы случайных величин $X_{k}, \ldots, X_{(k+l-1)\left(n_{j}\right)}$ и $X_{(k+l+m)\left(n_{j}\right)}, \ldots, X_{(k+l+m+s-1)\left(n_{j}\right)}$ независимы при всех $k=0, \ldots, n_{j}, l, s \geq 1$.

3. Пусть совместное распределение случайных величин $X_{i_{1}}^{(j)}, \ldots, X_{i_{k}}^{(j)}$ инвариантно относительно циклического сдвига, то есть закон распределения набора случайных величин $X_{i_{1}}^{(j)}, \ldots, X_{i_{k}}^{(j)}$ при $0 \leq i_{1}<i_{2}<\ldots<i_{k} \leq n_{j}-1$ совпадает с распределением набора $X_{\left(i_{1}+h\right)\left(n_{j}\right)}^{(j)}, \ldots, X_{\left(i_{k}+h\right)\left(n_{j}\right)}^{(j)}$, где $h \in \mathbb{Z}$.

Определим величины $\delta^{(j)}, \delta_{0}^{(j)}, \delta_{1}^{(j)}, j=1, \ldots, r$, равенствами

$$
v_{a_{2} a_{1}}^{(j)}=\frac{1}{4}\left(n_{j}+(-1)^{a_{1}} \delta^{(j)}+2(-1)^{a_{2}} \delta_{a_{1}}^{(j)}\right),
$$

где $v_{a_{2} a_{1}}^{(j)}$ - число знаков в $X^{(j)}$, двоичная запись которых равна $a_{2} a_{1}, a_{1}, a_{2} \in\{0,1\}$. Обозначим $v_{a_{2} a_{1}}^{(1, \ldots, r)}$ число знаков в $Z_{0} \ldots, Z_{T-1}$, имеющих двоичную запись $a_{2} a_{1}$, $a_{1}, a_{2} \in\{0,1\}$. В работе [2] показано, что

$$
v_{a_{2} a_{1}}^{(1, \ldots, r)}=\frac{1}{4}\left(n_{1} \ldots n_{r}+(-1)^{a_{1}} \delta^{(1, \ldots, r)}+2(-1)^{a_{2}} \delta_{a_{1}}^{(1, \ldots, r)}\right)
$$

где $\delta^{(1, \ldots, r)}=\delta^{(1)} \ldots \delta^{(r)}, \delta_{0}^{(1, \ldots, r)}+\mathrm{i} \delta_{1}^{(1, \ldots, r)}=\prod_{j=1}^{r}\left(\delta_{0}^{(j)}+\mathrm{i} \delta_{1}^{(j)}\right)$.

Нас интересует предельный при $n_{1}, \ldots, n_{r} \rightarrow \infty$ закон распределения случайного вектора $\vec{v}^{(1, \ldots, r)}=\left(v_{00}^{(1, \ldots, r)}, \ldots, v_{11}^{(1, \ldots, r)}\right)^{T}$. Ясно, что эта задача эквивалентна задаче о предельном законе распределения случайного вектора $\nabla^{(1, \ldots, r)}=\left(\delta^{(1, \ldots, r)}, \delta_{0}^{(1, \ldots, r)}, \delta_{1}^{(1, \ldots, r)}\right)^{T}$.

Переходим к изложению основного результата работы. Пусть

$$
\begin{gathered}
\Sigma^{(j)}=\left(\begin{array}{cccc}
\sigma_{0}^{(j)^{2}} & \rho_{01}^{(j)} \sigma_{0}^{(j)} \sigma_{1}^{(j)} & \rho_{02}^{(j)} \sigma_{0}^{(j)} \sigma_{2}^{(j)} & \rho_{03}^{(j)} \sigma_{0}^{(j)} \sigma_{3}^{(j)} \\
\rho_{01}^{(j)} \sigma_{0}^{(j)} \sigma_{1}^{(j)} & \sigma_{1}^{(j)^{2}} & \rho_{12}^{(j)} \sigma_{1}^{(j)} \sigma_{2}^{(j)} & \rho_{13}^{(j)} \sigma_{1}^{(j)} \sigma_{3}^{(j)} \\
\rho_{02}^{(j)} \sigma_{0}^{(j)} \sigma_{2}^{(j)} & \rho_{12}^{(j)} \sigma_{1}^{(j)} \sigma_{2}^{(j)} & \sigma_{2}^{(j)^{2}} & \rho_{23}^{(j)} \sigma_{2}^{(j)} \sigma_{3}^{(j)} \\
\rho_{03}^{(j)} \sigma_{0}^{(j)} \sigma_{3}^{(j)} & \rho_{13}^{(j)} \sigma_{1}^{(j)} \sigma_{3}^{(j)} & \rho_{23}^{(j)} \sigma_{2}^{(j)} \sigma_{3}^{(j)} & \sigma_{3}^{(j)^{2}}
\end{array}\right), \\
\sigma_{2 a_{2}+a_{1}}^{(j) 2}=\frac{3}{16}-\frac{m}{8}+2 \sum_{0<k^{\prime} \leq m} \mathbf{P}\left\{X_{0}^{(j)}=2 a_{2}+a_{1}, X_{k^{\prime}}^{(j)}=2 a_{2}+a_{1}\right\}, \\
\rho_{2 a_{2}+a_{1}, 2 b_{2}+b_{1}}^{(j)}=\frac{-1-2 m+32 \sum_{0<k^{\prime} \leq m} \mathbf{P}\left\{X_{0}^{(j)}=2 a_{2}+a_{1}, X_{k^{\prime}}^{(j)}=2 b_{2}+b_{1}\right\}}{16 \sigma_{2 a_{2}+a_{1}}^{(j)} \sigma_{2 b_{2}+b_{1}}^{(j)}},
\end{gathered}
$$

где $a_{1}, a_{2}, b_{1}, b_{2} \in\{0,1\},\left(a_{2}, a_{1}\right) \neq\left(b_{2}, b_{1}\right)$.

Положим

$$
\Sigma_{\tilde{\nabla}^{(j)}}=C \Sigma^{(j)} C^{T}, C=\left(\begin{array}{cccc}
2 & 0 & 2 & 0 \\
1 & 0 & -1 & 0 \\
0 & 1 & 0 & -1
\end{array}\right) .
$$


Теорема 11. Пусть выполнены условия 1,2 и 3, при всех $j$ матрицы $\sum_{\tilde{r}(j)}$ невырождены. Если параметры $m$ и $r$ үิ фиксированы, а все $n_{j} \rightarrow \infty, \quad \sigma_{k}^{(j)}=O(1)$, то случайный вектор

$$
\tilde{\nabla}^{(1, \ldots, r)}=\left(n_{1} \ldots n_{r}\right)^{-1 / 2}\left(\delta^{(1, \ldots, r)}, \delta_{0}^{(1, \ldots, r)}, \delta_{1}^{(1, \ldots, r)}\right)^{T}
$$

сходится по распределению к случайному вектору $\left(\eta, \eta_{0}, \eta_{1}\right)^{T}$, где

$$
\eta=\prod_{j=1}^{r} \eta^{(j)}, \eta_{0}+\mathrm{i} \eta_{1}=\prod_{j=1}^{r}\left(\eta_{0}^{(j)}+\mathrm{i} \eta_{1}^{(j)}\right),
$$

случайные векторы $\left(\eta^{(j)}, \eta_{0}^{(j)}, \eta_{1}^{(j)}\right)$ независимы между собой и распределены по нормальному закону с нулевыми средними и ковариационными матрицами $\Sigma_{\tilde{\bar{\nabla}}(j)}$.

Замечание 1. Сравним полученный результат для зависимых заполнений с результатом работы [4]. В ней показано, что при независимых заполнениях регистров $X^{(1)}, \ldots, X^{(r)}$ закон распределения случайного вектора $\tilde{\nabla}^{(1, \ldots, r)}$ сходится к распределению того же вектора $\left(\eta, \eta_{0}, \eta_{1}\right)^{T}$, но в образующих его наборах $\left(\eta^{(j)}, \eta_{0}^{(j)}, \eta_{1}^{(j)}\right)$ случайные величина $\eta^{(j)}$ и вектор $\left(\eta_{0}^{(j)}, \eta_{1}^{(j)}\right)$ независимы между собой. За счет этого удается написать несколько более простое выражение для предельного распределения, основанное на переходе в полярную систему координат.

Замечание 2. Если при всех $j=1, \ldots, r$

$$
\begin{aligned}
\sigma_{0}^{(j)} & =\sigma_{2}^{(j)}, \sigma_{0}^{(j)}\left(\rho_{01}^{(j)} \sigma_{1}^{(j)}-\rho_{03}^{(j)} \sigma_{3}^{(j)}\right)= \\
& =\sigma_{2}^{(j)}\left(\rho_{23}^{(j)} \sigma_{3}^{(j)}-\rho_{12}^{(j)} \sigma_{1}^{(j)}\right),
\end{aligned}
$$

то ковариационные матрицы $\Sigma_{\tilde{y}^{(j)}}$ будут иметь такой же вид, как в теореме 2 работы [2], которая была доказана для случая независимых и равновероятных знаков внутри каждого регистра. При выполнении условий предельный закон распределения из теоремы 1 совпадает с предельным законом, приведенным в теореме 2 работы [2].

\section{Доказательства}

Доказательство теоремы 1. Начнем с того, что выпишем первые два момента случайного вектора $\vec{v}^{(j)}=\left(v_{00}^{(j)}, \ldots, v_{11}^{(j)}\right)^{T}$.

Лемма 11. Пусть выполнены условия 1 , 2 и 3. Тогда

$$
\mathbf{E} \overrightarrow{\boldsymbol{v}}^{(j)}=\frac{n_{j}}{4}\left(\begin{array}{llll}
1 & 1 & 1 & 1
\end{array}\right)^{T}, \Sigma_{\overrightarrow{\mathrm{v}}^{(j)}}=n_{j} \Sigma^{(j)} .
$$

Из формулы следует, что $\delta^{(j)}=2 v_{0}^{(j)}-n_{j}, \delta_{a}^{(j)}=v_{0 a}^{(j)}-v_{1 a}^{(j)}, a \in\{0,1\}$.

Рассмотрим вектор

$$
\nabla^{(j)}=\left(\delta^{(j)}, \delta_{0}^{(j)}, \delta_{1}^{(j)}\right)^{T} .
$$

Так как

$$
\nabla^{(j)}=C \vec{v}^{(j)}-\left(n_{j}, 0,0\right)^{T},
$$

то $\mathbf{E} \nabla^{(j)}=\overrightarrow{0}, \Sigma_{\nabla^{(j)}}=C \Sigma_{\vec{v}^{(j)}} C^{T}$.

Следствие 1. Пусть выполнены условия 1, 2 и 3 . Тогда случайный вектор $\tilde{\nabla}^{(j)}=n_{j}^{-1 / 2}\left(\delta^{(j)}, \delta_{0}^{(j)}, \delta_{1}^{(j)}\right)^{T} \quad$ имеет нулевое среднее и ковариационную матрицу $\Sigma_{\tilde{\gamma}^{(j)}}$. Лемма 22. Пусть выполнены условия теоремы 1. Тогда случайный вектор $\tilde{\nabla}^{(j)}=n_{j}^{-1 / 2}\left(\delta^{(j)}, \delta_{0}^{(j)}, \delta_{1}^{(j)}\right)^{T} \quad$ асимптотически нормален с нулевым средним и ковариационной матрицей $\Sigma_{\tilde{\nabla}^{(j)}}$.

Доказательство леммы 1. Сначала выпишем первые два момента величины $v_{a_{2} a_{1}}^{(j)}$. Так как $v_{a_{2} a_{1}}^{(j)}=\sum_{k=0}^{n_{j}-1} I\left\{X_{k}^{(j)}=2 a_{2}+a_{1}\right\}$, то

$$
\mathbf{E} v_{a_{2} a_{1}}^{(j)}=\sum_{k=0}^{n_{j}-1} \mathbf{P}\left\{X_{k}^{(j)}=2 a_{2}+a_{1}\right\}=\frac{n_{j}}{4}
$$

$$
\begin{gathered}
\mathbf{D} \mathbf{v}_{a_{2} a_{1}}^{(j)}=\mathbf{D}\left(\sum_{k=0}^{n_{j}-1} I\left\{X_{k}^{(j)}=2 a_{2}+a_{1}\right\}\right)= \\
=\sum_{k=0}^{n_{j}-1} \mathbf{D} I\left\{X_{k}^{(j)}=2 a_{2}+a_{1}\right\}+2 \sum_{k=0}^{n_{j}-1} \sum_{k^{\prime}=k+1}^{n_{j}-1} \operatorname{cov}\left(I\left\{X_{k}^{(j)}=2 a_{2}+a_{1}\right\}, I\left\{X_{k^{\prime}}^{(j)}=2 a_{2}+a_{1}\right\}\right)= \\
=\frac{3 n_{j}}{16}+2 \sum_{k=0}^{n_{j}-1} \sum_{0<k^{\prime}-k \leq m}\left(\mathbf{P}\left\{X_{k}^{(j)}=2 a_{2}+a_{1}, X_{k^{\prime}}^{(j)}=2 a_{2}+a_{1}\right\}-\frac{1}{16}\right)= \\
=\frac{3 n_{j}}{16}+2 n_{j} \sum_{1 \leq k^{\prime} \leq m}\left(\mathbf{P}\left\{X_{0}^{(j)}=2 a_{2}+a_{1}, X_{k^{\prime}}^{(j)}=2 a_{2}+a_{1}\right\}-\frac{1}{16}\right)= \\
=n_{j}\left(\frac{3}{16}-\frac{m}{8}+2 \sum_{0<k^{\prime} \leq m} \mathbf{P}\left\{X_{0}^{(j)}=2 a_{2}+a_{1}, X_{k^{\prime}}^{(j)}=2 a_{2}+a_{1}\right\}\right) .
\end{gathered}
$$


Кроме того, при $\left(a_{2}, a_{1}\right) \neq\left(b_{2}, b_{1}\right)$

$$
\begin{gathered}
\operatorname{cov}\left(v_{a_{2} a_{1}}^{(j)}, v_{b_{2} b_{1}}^{(j)}\right)=\operatorname{cov}\left(\sum_{k=0}^{n_{j}-1} I\left\{X_{k}^{(j)}=2 a_{2}+a_{1}\right\}, \sum_{k=0}^{n_{j}-1} I\left\{X_{k^{\prime}}^{(j)}=2 b_{2}+b_{1}\right\}\right)= \\
=\sum_{k=0}^{n_{j}-1 n_{j}-1} \sum_{k^{\prime}=0} \operatorname{cov}\left(I\left\{X_{k}^{(j)}=2 a_{2}+a_{1}\right\}, I\left\{X_{k^{\prime}}^{(j)}=2 b_{2}+b_{1}\right\}\right)= \\
=-\frac{n_{j}}{16}+2 \sum_{k=0}^{n_{j}-1} \sum_{1 \leq k^{\prime}-k \leq m}\left(\mathbf{P}\left\{X_{k}^{(j)}=2 a_{2}+a_{1}, X_{k^{\prime}}^{(j)}=2 b_{2}+b_{1}\right\}-\frac{1}{16}\right)= \\
=-\frac{n_{j}}{16}+2 n_{j} \sum_{1 \leq k^{\prime} \leq m}\left(\mathbf{P}\left\{X_{0}^{(j)}=2 a_{2}+a_{1}, X_{k^{\prime}}^{(j)}=2 b_{2}+b_{1}\right\}-\frac{1}{16}\right)= \\
=n_{j}\left(-\frac{1}{16}-\frac{m}{8}+2 \sum_{1 \leq k^{\prime} \leq m} \mathbf{P}\left\{X_{0}^{(j)}=2 a_{2}+a_{1}, X_{k^{\prime}}^{(j)}=2 b_{2}+b_{1}\right\}\right) .
\end{gathered}
$$

Формулы следуют из равенств - Лемма 1 доказана.

Доказательство леммы 2. Согласно теореме 1 п. 4 § 13 гл. 2 книги [3] достаточно показать, что любая линейная комбинация $W=\alpha \delta^{(j)}+\beta_{0} \delta_{0}^{(j)}+\beta_{1} \delta_{1}^{(j)}, \alpha^{2}+\beta_{0}^{2}+\beta_{1}^{2}>0$, асимптотически нормальна. Так как вектор $\nabla^{(j)}$ получен в результате линейного преобразования $\vec{v}^{(j)}$, то вместо случайной величины $W$ можно рассмотреть случайную величину

$$
\tilde{W}=\alpha_{0} v_{00}^{(j)}+\alpha_{1} v_{01}^{(j)}+\alpha_{2} v_{10}^{(j)}+\alpha_{3} v_{11}^{(j)} .
$$

Сначала вычислим числовые характеристики суммы $\tilde{W}$ :

$$
\begin{aligned}
\mathbf{E} \tilde{W}=\left(\alpha_{0}+\alpha_{1}+\alpha_{2}+\alpha_{3}\right) \frac{n_{j}}{4}, & \begin{array}{c}
\text { но. Если существует така } \\
\mathbf{P}\left\{\left|\xi_{v}-E \xi_{v}\right| \leq B\right\}=1 \text { для л }
\end{array} \\
& \left|\mathbf{P}\left\{\frac{W-\mathbf{E} W}{\sqrt{\mathbf{D} W}}<x\right\}-\Phi(x)\right| \leq 32(1+\sqrt{6})|V| D^{2} B^{3}(\mathbf{D} W)^{-3 / 2}
\end{aligned}
$$

где $W=\sum_{v \in V} \xi_{v}, D$ - максимальная степень вершины в графе $G$.

Согласно определению

$$
\tilde{W}=\alpha_{0} \sum_{k=0}^{n_{j}-1} I\left\{X_{k}^{(j)}=0\right\}+\alpha_{1} \sum_{k=0}^{n_{j}-1} I\left\{X_{k}^{(j)}=1\right\}+\alpha_{2} \sum_{k=0}^{n_{j}-1} I\left\{X_{k}^{(j)}=2\right\}+\alpha_{3} \sum_{k=0}^{n_{j}-1} I\left\{X_{k}^{(j)}=3\right\} .
$$

Пусть $U=\left\{u=(a, k): k=0, \ldots, n_{j}-1 ; a=0, \ldots, 3\right\}$. Положим

Тогда

$$
I_{u}=\alpha_{a} I\left\{X_{k}^{(j)}=a\right\}, u=(a, k) \in U .
$$

$$
\tilde{W}=\sum_{u \in U} I_{u}
$$


Таким образом, к случайной величине $\tilde{W}$ применима оценка . Граф зависимостей слагаемых суммы имеет множество вершин $U$. Ясно, что в качестве константы $B$ можно взять $B=\max _{a=0, \ldots 3}\left|\alpha_{a}\right|$. Вершины

$$
\left|\mathbf{P}\left\{\frac{\tilde{W}-\mathbf{E} \tilde{W}}{\sqrt{\mathbf{D} \tilde{W}}}<x\right\}-\Phi(x)\right| \leq 32(1+\sqrt{6}) 4 n_{j}(2 m+1)^{2}\left(\max _{a=0, \ldots, 3}\left|\alpha_{a}\right|\right)^{3}(\mathbf{D} \tilde{W})^{-3 / 2}=O\left(n_{j}^{-1 / 2}\right) .
$$

Таким образом, закон распределения случайной величины $\tilde{W}$ асимптотически нормален. Лемма 2 доказана.

Из следствия 1 , леммы 2, формулы и независимости наборов $X^{(1)}, \ldots, X^{(r)}$ следует утверждение теоремы. Теорема 1 доказана.

\section{Заключение}

В работе изучены свойства мультициклической случайной последовательности, образованной независимы между собой регистрами, при этом случайные величины в каждом регистре имеют равновероятные одномерные распределения, но m-зависимы по кругу, а их распределение инвариантно относительно циклического сдвига. Для вектора из чисел появлений знаков $\{0,1,2,3\}$ на полном цикле мультициклической последовательности доказана многомерная предельная теорема нормального типа в случае, когда длины регистров стремятся к бесконечности, а их число $\mathrm{r}$ остается фиксированным. Изучен вопрос $u=(a, k) \quad$ и $\quad u^{\prime}=\left(a^{\prime}, k^{\prime}\right), \quad u, u^{\prime} \in U$, соединены ребрами, если $\left|k-k^{\prime}\right| \leq m$. Значит, $D=2 m+1$.

$n_{j} \rightarrow \infty$

Так как $|U|=4 n_{j}$, то из и получим при

об устойчивости соответствующего предельного распределения для случая независимых случайных величин, заполняющих регистры (генератора Пола).

Работа выполнена при финансовой поддержке Министерства образования и науки РФ (тема 1.2640.2014).

\section{Список литературы}

1. Меженная Н.М., Михайлов В.Г. О распределении числа единиц в выходной последовательности генератора Пола над полем GF(2) // Математические вопросы криптографии. - 2013. - № 4, вып. 4. - С. 95-107.

2. Меженная Н.М., Михайлов В.Г. О числе появлений знаков в мультициклической случайной последовательности по модулю 4 // Дискретная математика. - 2014. - т. 26, вып. 4. - С. 51-58.

3. Ширяев А.Н. Вероятность. - М.: Наука, 1989. - 581 с.

4. Buldi P., Rinott Y. On normal approximations of distributions in terms of dependency graph // Ann. Probab. 1989. - v. 17, № 5. - P. 1646-1650.

5. Mezhennaya N.M. Convergence rate estimators for the number of ones in outcome sequence of MCV generator with $\mathrm{m}$-dependent registers items // Siberian Electronic Mathematical Reports. - 2014. - v. 11. - P. 18-25.

6. Pohl P. Description of MCV, A pseudo-random number generator // Scand. Actuarial J. - 1976. - v. 1. - P. 1-14. 